\title{
A Well Clear Recommendation for Small UAS in High-Density, ADS-B-Enabled Airspace
}

Timothy McLain

Department of Mechanical Engineering, Brigham Young University, mclain@byu.edu

Matthew O. Duffield

Department of Mechanical Engineering, Brigham Young University

Follow this and additional works at: https://scholarsarchive.byu.edu/facpub

Part of the Mechanical Engineering Commons

\section{Original Publication Citation}

Duffield, M. and McLain T. A Well Clear Recommendation for Small UAS in High-Density, ADS-BEnabled Airspace, Proceedings of the AIAA Infotech@Aerospace Conference, AIAA 2017-0908, January 2017, Grapevine, Texas.

\section{BYU ScholarsArchive Citation}

McLain, Timothy and Duffield, Matthew O., "A Well Clear Recommendation for Small UAS in High-Density, ADS-B-Enabled Airspace" (2017). Faculty Publications. 1877.

https://scholarsarchive.byu.edu/facpub/1877 


\title{
A Well Clear Recommendation for Small UAS in High-Density, ADS-B-Enabled Airspace
}

\author{
Matthew O. Duffield* and Timothy W. McLain ${ }^{\dagger}$ \\ Brigham Young University, Provo, UT, 84606, USA
}

\begin{abstract}
With the growing popularity of small unmanned aircraft systems (UAS), there is a significant need to enable small UAS to detect and avoid collisions with both manned and unmanned aircraft. The capabilities of ADS-B make it an attractive sensor for detect and avoid (DAA), but it is susceptible to frequency congestion. This paper quantitatively analyzes the frequency limitations of $978 \mathrm{MHz}$ ADS-B. It then uses these limitations to make a recommendation for well clear in ADS-B-equipped airspace that has a high density of small UAS operations.
\end{abstract}

\section{Introduction}

The number of applications of unmanned aircraft systems (UAS) is growing at a significant pace. Consequently the need for UAS in the National Airspace System is compounding at a similar rate. To fill this public and private demand for UAS operation, a vast number of companies continue to invest in, and build around, UAS technology. The demand for UAS operations is manifest by the hundreds petitions to allow UAS operations under Section 333 of the FAA Modernization and Reform Act of 2012 [1]. In laying the foundation for a long-term solution for UAS in the NAS, the Federal Aviation Administration (FAA) has mandated that UAS be capable of an equivalent level of safety (ELOS) to the see-and-avoid mandate for manned aircraft $[2,3]$. This mandate has come to be known as detect and avoid (DAA).

As a result of the large number of potential UAS applications, the rapidly developing DAA capability on UAS, and the expected integration of UAS into the NAS, future NAS conditions will include high-volume UAS operations. Numerous logistics companies such as Amazon, UPS, and DHL have announced plans to use UAS to deliver packages. Several restaurants and bakeries have indicated that they plan to use UAS to provide rapid delivery to their customers [4]. Considering the wide array of other potential UAS applications, it is likely that there may be hundreds, or even thousands, of small UAS operating over highly populated areas. Such a high volume of UAS presents a new and unique air traffic control (ATC) challenge. Current ATC definitions of safe speeds and separation distances between aircraft are based on manned aircraft characteristics, which are much larger, fly much faster, and operate at higher altitudes than small UAS. To ensure safe and efficient UAS operation, DAA capability must be capable of handling high-density UAS operations. Ultimately integrating the anticipated high volume of small UAS into the NAS is a challenging aspect of both DAA and ATC development.

\section{Background}

The goal of DAA is to ensure that UAS are able to avoid other manned and unmanned aircraft. This very broad definition can be broken down into two different levels: self-separation assurance and collision avoidance. In self-separation assurance the objective is to remain well clear of other aircraft. Well clear is a term used in FAA-defined visual flight rules (VFR) regulations, and it is a qualitatively-defined safe, comfortable distance from other aircraft [3]. Pilots flying under VFR regulations determine their own well clear distance for each encounter with another aircraft. To maintain self-separation, aircraft make small, gradual path adjustments well in advance of a collision. In the event that an intruder penetrates the well

*Graduate Student, Mechanical Engineering, Student Member.

${ }^{\dagger}$ Professor, Mechanical Engineering, Associate Fellow. 
clear volume of an ownship, it is referred to as a conflict, or in other words an unsafe, but non-catastrophic encounter. In a collision avoidance scenario, on the other hand, a near-mid-air collision (NMAC) is imminent, and the goal is to rapidly and aggressively avoid the collision. A collision is typically, although not officially, defined as two aircraft being within $200 \mathrm{ft}$ in altitude and $500 \mathrm{ft}$ horizontally [5]. Should two aircraft fail to maintain at least this much distance between them it is referred to as an NMAC and is a serious event.

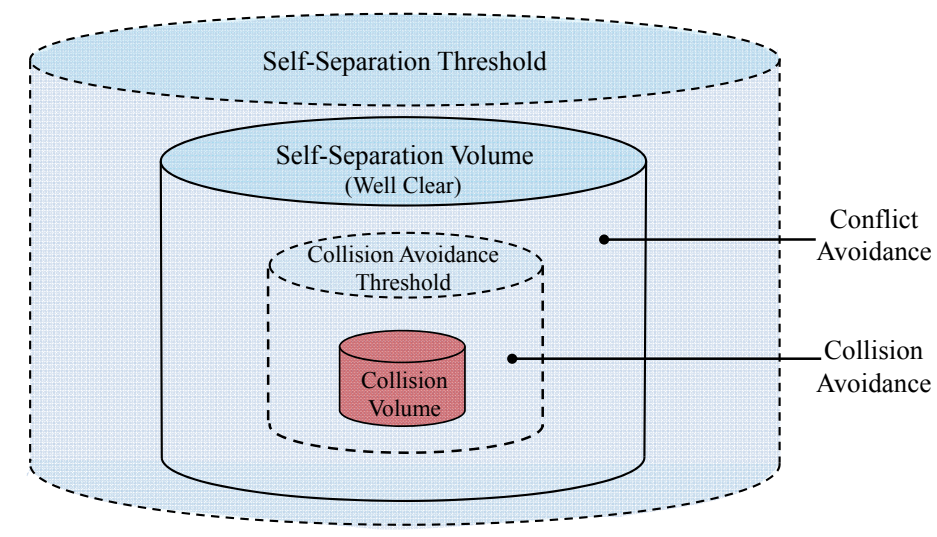

Figure 1. DAA volumes that indicate DAA levels.

Figure 1 shows the DAA volumes graphically. The figure is not drawn to scale, but it does show the relationship between the self-separation, or well clear, volume and the collision volume. Also Figure 1 shows the self-separation threshold and collision avoidance threshold. These thresholds are the point at which the ownship begins to maneuver to maintain the well clear or collision volume respectively. In other words, an aircraft is not considered to be an intruder until it crosses the self-separation threshold (SST). When the aircraft does cross the SST, then the ownship begins to maneuver to maintain well clear (WC). Similarly, an intruder is not considered to be a threat until it crosses the collision avoidance threshold (CAT). Once it crosses the CAT, the ownship begins to maneuver to prevent the intruder from entering the collision volume of the ownship. This set of volumes and thresholds creates an important framework for determining sensor requirements, collision prediction methods, and collision avoidance ability.

To allow UAS to successfully achieve an ELOS to manned aircraft operations, UAS must be capable of maintaining well clear from all other aircraft in a similar way to that of manned aircraft. By emphasizing self-separation and constantly maintaining well clear from other aircraft, the ability and probability of a UAS preventing collisions increases dramatically. Focusing exclusively on collision avoidance, results in the UAS having to make aggressive, reactive, and last-minute maneuvers. In many cases these types of maneuvers push the computational, and physical limits of the UAS. By focusing on self-separation, there is a much longer reaction time available for a UAS to identify an intruder, predict its path, and execute an avoidance maneuver. This results in a much higher level of safety.

Focusing on self-separation highlights several challenges associated with maintaining well clear. The sensor capabilities necessary to detect intruders at long ranges can be difficult to achieve on board a small UAS. Long-range path planning can pose a significant computational challenge, especially for a power and space-limited small UAS. Finally autonomous self-separation capability requires that there be a quantitative definition of well clear, which does not currently exist. Each of these challenges poses an exciting opportunity in the development of small UAS.

\section{A. Current Sensor Technologies}

For small UAS, those weighing less than fifty-five pounds, the algorithms and hardware necessary for DAA make up a notable portion of the size, weight, and power (SWaP) resources available. Traditional aircraftdetecting sensors are designed for manned aircraft that offer a significantly larger payload and much more powerful electrical system than those available on small UAS. Scaling traditional sensors down to small UAS sizes often requires compromises in range, accuracy, field of view, or processing speed. Such compromises can reduce the overall capability of the DAA system and consequently decrease the assurance of collision prevention. Significant efforts have gone into overcoming these challenges and identifying sensors suitable 
for DAA on small UAS. For radar and cameras, recent developments have resulted in notable improvements, but current capabilities are not yet suitable for small UAS [6].

Automatic Dependent Surveillance-Broadcast (ADS-B), however, is a cooperative sensor that is a promising option for DAA on small UAS. It has been demonstrated in small UAS flight testing to have an omnidirectional range of $20 \mathrm{nmi}$ [7], and due to the fact that the cooperative information is shared over radio waves it is relatively unaffected by adverse weather conditions. An omni-directional antenna and low power requirements for both transponder and receiver hardware contribute to the promising characteristics of ADSB. Two major drawbacks of ADS-B are its fundamentally cooperative nature and its bandwidth limitations. The cooperative aspect of ADS-B requires widespread adoption of ADS-B technology to ensure detect and avoid reliability. While the Federal Aviation Administration does not yet require all aircraft to be equipped with ADS-B transponders, the 2020 mandate requiring all aircraft in A, B, C, and some E class airspace to equip with ADS-B [8] is a significant step. Due to the fact that ADS-B messages are sent using a finitecapacity multiple access protocol, there is limited bandwidth. If too many aircraft are transmitting on ADS-B at the same time, then the transmissions may interfere with each other and become unusable. While this is not likely to be a concern for current NAS conditions, a future environment with many small UAS operating in close proximity may encounter bandwidth limitations. Even with its cooperative nature and possible bandwidth limitations, ADS-B is a promising sensor for self-separation DAA efforts.

\section{B. The Need To Define Well Clear}

To allow UAS to autonomously maintain self-separation, both a quantitative well clear definition and selfseparation threshold are necessary. Typically SST and WC definitions are defined in one of three ways: the distance between the ownship and intruder, the time to collision or closest point of approach, or a combination of both distance and time metrics. Joint definitions are the most common. The distance-based definitions are quite intuitive. Each aircraft is surrounded by a safety volume, typically a cylinder, such that any intruder that penetrates that volume is said to have lost self-separation. The time-based definitions are also intuitive, but more difficult to visualize. For each intruder, the ownship calculates a time metric, such as the time to closest point of approach. If this time is less than a given threshold then self-separation, or well clear, has been lost. There are many different time metrics that strive to estimate when two aircraft will collide [9].

Recent literature contains several proposed self-separation threshold and well clear definitions [10-14]. Johnson, Mueller, and Santiago detail and compare several of these different definitions [10]. Additionally, they provide a valuable insight into the application of SST and WC definitions by using recorded VFR traffic and simulated UAS to map time-based well clear definitions onto a distance-from-ownship plot [10]. Such a mapping demonstrates the range at which the time-based SST and WC definitions are enacted. Interestingly in the majority of cases, the time-based definition was reached long before the distance-based definition. The result of their simulations led them to recommend that DAA sensors be capable of sensing intruders $12 \mathrm{nmi}$ in front of and $5 \mathrm{nmi}$ behind an ownship to achieve the proposed SST and WC definitions. While their contribution is valuable, it, along with the other existing well clear recommendations, was built using medium to large UAS in airspace with manned aircraft. A need exists to define well clear for small, hand-launch type, UAS in low altitude airspace.

Currently, one of the primary small-UAS-suitable sensors capable of the recommended range is ADS-B. While ADS-B does offer a promising sensor for self-separation, it is susceptible to frequency congestion. In environments with a large number of ADS-B-equipped aircraft it is possible for transmitted information to interfere with other ADS-B transmissions. The development and validation efforts used to define the currently proposed SST and WC definitions do not take into account congestion of the ADS-B frequency. It is necessary to investigate the effects of ADS-B congestion on the self-separation threshold and well clear definitions and propose a well clear definition for small UAS in congested ADS-B airspace.

The objective of this paper is to present a well clear definition for small UAS in high-density, ADS-Bdependent airspace.

\section{Approach}

To define a self-separation threshold and well clear definition for small UAS, it is necessary to understand the bandwidth limitations of ADS-B and the capability of the small UAS. The analysis of ADS-B bandwidth 
limitations presented here will focus on the $978 \mathrm{MHz}$ frequency. This frequency is intended for aircraft operating under $10000 \mathrm{ft}$. As such it is reasonable that the vast majority, if not all, small UAS will equip with the $978 \mathrm{MHz}$ frequency. It is important to note that in our approach we assume that the ADS-B measurements from the intruder aircraft are filtered using an estimator such as a Kalman filter. Although not a primary result, the benefit of such estimation is demonstrated.

\section{A. ADS-B Bandwidth Limitations}

1. ADS-B Multiple Access Scheme

To support ADS-B messages from multiple transmitters in the same second, the $978 \mathrm{MHz}$ ADS-B frequency uses a time-division multiple access (TDMA) scheme. In other words, each ADS-B message is intended to be transmitted in its own time slot. The Universal Access Transceiver (UAT) message is divided up into two major time sections: the ground uplink message portion and the traffic transmission portion. The combination of these two sections is referred to as a UAT frame. Each frame consumes 1 second [15]. With in the traffic transmission portion multiple distinct message start opportunities (MSOs) where in individual aircraft can transmit their ADS-B message [6]. While the TDMA scheme does permit some interference, the goal of the method is to prevent two aircraft from systematically interfering with each other. For a large number of aircraft, however, it is possible that ADS-B messages will interfere with each other much more than was intended.

\section{Probability of Interference at a Single Time Step}

For the TDMA structure of the $978 \mathrm{MHz}$ frequency, it is possible to calculate a probability of interference. Given a uniform distribution of the selection of the MSO, the probability that a given transmitter selects a given MSO can be simply determined. For two transmitters, the probability that the two transmitters select the same frequency can also be determined. Since each transmitter selects an MSO without any knowledge of other transmitter's selection, the two selections are independent. Thus the probability that they both select a given frequency is the product of the two probabilities that they individually select that frequency. Given 3200 MSOs the probability that transmitters A and B simultaneously select $\mathrm{MSO}_{i}$ is

$$
\begin{aligned}
\mathrm{P}\left(\mathrm{A}=\mathrm{MSO}_{i}\right) & =\frac{1}{3200} \\
\mathrm{P}\left(\mathrm{B}=\mathrm{MSO}_{i}\right) & =\frac{1}{3200} \\
\mathrm{P}\left(\mathrm{A}=\mathrm{B}=\mathrm{MSO}_{i}\right) & =\left(\frac{1}{3200}\right) \times\left(\frac{1}{3200}\right) \\
& =\frac{1}{3200^{2}} .
\end{aligned}
$$

Thus the probability that two transmitters simultaneously transmit on a given frequency is one over the number of MSOs squared. This, however, is only for a given $\mathrm{MSO}_{i}$. For the probability of interference at any MSO for a given second, we need $\mathrm{P}\left(\mathrm{A}=\mathrm{B}=\mathrm{MSO}_{1} \cup \mathrm{A}=\mathrm{B}=\mathrm{MSO}_{2} \cup \ldots \cup \mathrm{A}=\mathrm{B}=\mathrm{MSO}_{3200}\right)$. This can be calculated by using the equation for a union [16]. Due to the fact that the events $\mathrm{P}\left(\mathrm{A}=\mathrm{B}=\mathrm{MSO}_{i}\right)$ and $\mathrm{P}\left(\mathrm{A}=\mathrm{B}=\mathrm{MSO}_{j}\right)$ are mutually exclusive for all $i$ and $j$, the intersection of any and all of these events is equal to zero. Thus we are left with the summation

$$
\begin{aligned}
& \mathrm{P}\left(\mathrm{A}=\mathrm{B}=\mathrm{MSO}_{1} \bigcup \mathrm{A}=\mathrm{B}=\mathrm{MSO}_{2} \bigcup \ldots \bigcup \mathrm{A}=\mathrm{B}=\mathrm{MSO}_{3200}\right) \\
&= \mathrm{P}\left(\mathrm{A}=\mathrm{B}=\mathrm{MSO}_{1}\right)+\mathrm{P}\left(\mathrm{A}=\mathrm{B}=\mathrm{MSO}_{2}\right)+\ldots \\
&+\mathrm{P}\left(\mathrm{A}=\mathrm{B}=\mathrm{MSO}_{3200}\right) \\
&= 3200 \times \frac{1}{3200^{2}} \\
&= \frac{1}{3200}
\end{aligned}
$$

This is then the probability that two transmitters transmit on the same MSO at a given second, $\mathrm{P}(\mathrm{A}=\mathrm{B})$, causing interference between the two. 
Now consider a third and fourth transmitter, $\mathrm{C}$ and $\mathrm{D}$, where $\mathrm{P}(\mathrm{A}=\mathrm{B})=\frac{1}{3200}$ and $\mathrm{P}(\mathrm{A}=\mathrm{C})=\frac{1}{3200}$ and $\mathrm{P}(\mathrm{A}=\mathrm{D})=\frac{1}{3200}$. To find the probability that $\mathrm{A}$ interferes with either $\mathrm{B}$ or $\mathrm{C}$ or $\mathrm{D}$, we need the union of all the individual probabilities, $\mathrm{P}(\mathrm{A}=\mathrm{B} \cup \mathrm{A}=\mathrm{C} \cup \mathrm{A}=\mathrm{D})$. This is also given by

$$
\begin{aligned}
& \mathrm{P}(\mathrm{A}=\mathrm{B} \bigcup \mathrm{A}=\mathrm{C} \bigcup A=D) \\
&= \mathrm{P}(\mathrm{A}=\mathrm{B})+\mathrm{P}(\mathrm{A}=\mathrm{C})+\mathrm{P}(\mathrm{A}=\mathrm{D}) \\
&-\mathrm{P}(\mathrm{A}=\mathrm{B}) \mathrm{P}(\mathrm{A}=\mathrm{C})-\mathrm{P}(\mathrm{A}=\mathrm{B}) \mathrm{P}(\mathrm{A}=\mathrm{D})-\mathrm{P}(\mathrm{A}=\mathrm{C}) \mathrm{P}(\mathrm{A}=\mathrm{D}) \\
&+\mathrm{P}(\mathrm{A}=\mathrm{B}) \mathrm{P}(\mathrm{A}=\mathrm{C}) \mathrm{P}(\mathrm{A}=\mathrm{D})
\end{aligned}
$$

Since each of these probabilities is equal, we let $x=\mathrm{P}(\mathrm{A}=\mathrm{B})=\mathrm{P}(\mathrm{A}=\mathrm{C})=\mathrm{P}(\mathrm{A}=\mathrm{D})=\frac{1}{3200}$. Then Equation (3) can be given by $3 x-3 x^{2}+x^{3}$. This is then the expression for the probability that transmitter $\mathrm{A}$ is interfered with at one time-step for an environment with four transmitters.

Extending this result to more transmitters, it becomes apparent that the pattern continues. For $n$ transmitters, $\mathrm{T}$, given that the event $\mathrm{T}_{0}=\mathrm{T}_{i}$ is stated as $E_{i}$

$$
\begin{aligned}
\mathrm{P}(I) & =\mathrm{P}\left(E_{1} \bigcup E_{2} \bigcup \ldots \bigcup E_{n-1}\right) \\
& =\left(\begin{array}{c}
n-1 \\
1
\end{array}\right) x-\left(\begin{array}{c}
n-1 \\
2
\end{array}\right) x^{2}+\ldots+(-1)^{n-1}\left(\begin{array}{l}
n-1 \\
n-2
\end{array}\right) x^{n-2}+(-1)^{n}\left(\begin{array}{l}
n-1 \\
n-1
\end{array}\right) x^{n-1}
\end{aligned}
$$

where $\left(\begin{array}{c}n-1 \\ i\end{array}\right)$ is a combination. This is then the probability that a transmitter $\mathrm{T}_{0}$ will be interfered with for an environment with $n$ transmitters for a given second, $\mathrm{P}\left(I_{t_{i}}\right)$.

Using this formula, however, poses a computational challenge. The combination formula $\left(\begin{array}{l}n \\ k\end{array}\right)$ reaches a maximum when $k=\frac{n}{2}$. Thus for a large number of transmitters, $\left(\begin{array}{l}n-1 \\ \frac{n-1}{2}\end{array}\right)$ is a very large number. For numbers near to this value, computers are unable to represent the value accurately. Additionally it is also possible that the numbers cannot be represented at all. For example $\left(\begin{array}{l}56 \\ 28\end{array}\right)$ is one of the largest combinations that MATLAB can represent accurately, and $\left(\begin{array}{c}1029 \\ 515\end{array}\right)$ is one of the largest combinations that MATLAB can represent at all. Any combination larger than this value is recognized as infinity. Thus if the number of transmitters is greater than 1030 MATLAB cannot compute the probability.

We developed a method to mitigate this using Sterling's approximation for factorials to calculate the combination of large values. Given the combination formula as $\left(\begin{array}{l}n \\ r\end{array}\right)=\frac{n !}{r !(n-r) !}$, Sterling's approximation can be applied to rewrite it as

$$
\left(\begin{array}{l}
n \\
r
\end{array}\right) \approx \frac{\sqrt{2 \pi n}\left(\frac{n}{e}\right)^{n}}{\left(\sqrt{2 \pi r}\left(\frac{r}{e}\right)^{r}\right)\left(\sqrt{2 \pi(n-r)}\left(\frac{n-r}{e}\right)(n-r)\right)}
$$

Taking the $\log _{n}$ reduces the formula to be

$$
\begin{aligned}
\log _{n}\left(\begin{array}{l}
n \\
k
\end{array}\right) \approx & \frac{1}{2}+n-n \log _{n}(e)-\frac{1}{2} \log _{n}(r)-r \log _{n}\left(\frac{r}{e}\right) \\
& -\frac{1}{2} \log _{n}(2 \pi(n-r))-(n-r) \log _{n}\left(\frac{n-r}{e}\right)
\end{aligned}
$$

Using this formula the computation of very large combinations is possible. Due to the fact that Sterling's approximation is most accurate for large factorials, smaller factorials are best calculated by traditional methods. Also, at very large factorials, there is still some numerical instability in the method. Thus, it should be used with caution. That being said, the numerical instability is notably less than that of traditional combination methods. As a result it is sufficient for our calculations.

With Equations (4) and (6) it is possible to calculate the probability that a given transmitter is interfered with at single time step for a given number of transmitting aircraft.

\section{Probability of Trackability}

Using the probability of interference for a given transmitter at a single time step and several key characteristics of tracking/estimating methods, it is possible to determine the probability of starting and maintaining a track for any given aircraft. We refer to this probability as the probability of trackability. 
Prior to presenting the method to calculate the probability of trackability, it is necessary to identify several terms. To initialize a track with any estimator, it is necessary to receive a set number of measurements in a set amount of time. Since ADS-B messages do not require data association, a track can be formed from just two measurements. The maximum set size is the maximum number of measurements that can be missed between two received measurements and allow them to still form a track. In other words, if four measurements can be missed between two good measurements and the two good measurements can still be used to form a track, then the maximum set size is six. Thus two out of six messages must be received to form a track.

Another key term is the number of missed measurements to terminate a track. This is the maximum number of measurements, after a track already exists, that can be missed before the track is declared as invalid. This value is dependent upon the covariance of the track, but a nominal value can be determined by taking the initial track covariance and propagating it forward without measurement updates until the covariance becomes so large that the track is terminated. The number of times that the track was propagated into the future is the number of missed measurements to kill a track.

Due to the fact that the MSO for a given transmitter is selected on a random basis each second, the probability of interference for a given second is independent from the probability of interference for a different second. Using this fact, the probability that consecutive measurements are interfered with is $\mathrm{P}\left(I_{t_{1}} \cap I_{t_{2}}\right)=\mathrm{P}\left(I_{t_{1}}\right) \mathrm{P}\left(I_{t_{2}}\right)$. Assuming that the number of transmitting aircraft remains the same between the two consecutive seconds, the probability remains the same, and thus the probability simplifies to $\mathrm{P}\left(I_{t_{1}} \cap I_{t_{2}}\right)=\mathrm{P}\left(I_{t_{1}}\right)^{2}$. This can be extended to more than just two consecutive seconds. In the case of tracking/estimation of intruders, the maximum set size and the number of missed measurements to terminate a track dictate how many measurements can be missed before tracking becomes unreliable. For the case of the maximum set size, if two measurements are not received in that set then the track cannot be formed. Thus the probability of forming a track is $\mathrm{P}\left(T_{\text {form }}\right)=1-\mathrm{P}\left(I_{t_{1}}\right)^{\text {MaxSetSize-1 }}$. For the number of missed measurements to kill a track, at least one measurement must be received in the set. Thus the probability of maintaining a track is $\mathrm{P}\left(T_{\text {maintain }}\right)=1-\mathrm{P}\left(I_{t_{1}}\right)^{\text {NumMissMeastoKillTrack. }}$.

These two probabilities, the probability to form a track and the probability to maintain a track, determine the robustness to missed measurements of a given tracker for a given number of transmitting aircraft. The overall probability of trackability is the lesser of these two probabilities. If either a track cannot be formed reliably or a track cannot be maintained reliably then the tracker is unable to track reliably. Thus $\mathrm{P}(T)=\min \left(\mathrm{P}\left(T_{\text {form }}\right), P\left(T_{\text {maintain }}\right)\right)$. The metric that leads to the minimum probability is referred to as the limiting tracking variable (LTV).

\section{Key Parameters}

As previously shown the probability of trackability is a function of many variables, but it can be reduced to a function of two variables. The full list of fundamental variables for the probability of trackability includes range, aircraft density, intruder speed, filter gate size, track covariance gate size, time between measurements, filtering method. Varying any of these parameters results in a change in the probability of trackabaility. These variables, however, can be formulated into two variables that fully describe the probability of trackability. The range and density combine to determine the total number of aircraft with in the visible region. The speed of the intruder, time between measurements, filter matrices, the Mahalanobis gate size, and track covariance gate size are necessary only to determine the maximum set size and the number of measurements necessary to kill a track. As previously stated, the smaller value between maximum set size and the number missed measurements to kill a track is the LTV. Thus the probability of trackability is actually a function of only the number of aircraft and the probability of trackability.

\section{B. Well Clear Definition Analysis}

For high-density airspace with a large number of small UAS, the possibility of ADS-B interference presents a unique challenge to maintaining well clear. As explained previously, maintaining WC requires detection of intruders at long-ranges. ADS-B message interference can limit the capability of ADS-B to detect intruders. Thus it is necessary to examine the the proposed self-separation threshold and well clear definitions from a system-design perspective to determine whether such definitions are suitable and achievable for current airspace regulations and anticipated small UAS densities. The method presented here determines a maximum 
and minimum achievable well clear distance and time. Those bounds can then be used to determine an appropriate well clear definition.

\section{Upper WC Bound Analysis Method}

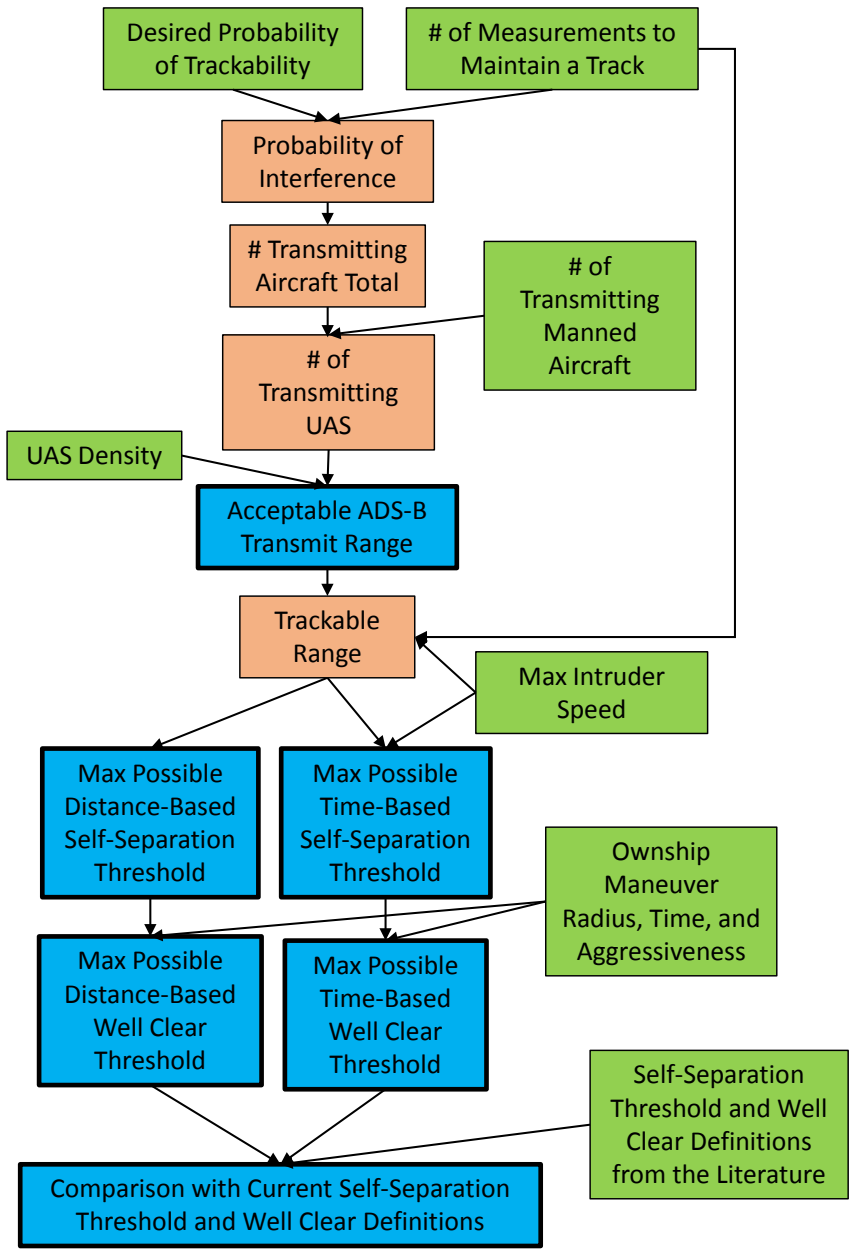

Figure 2. Variables and method by which SST and WC definitions can be evaluated.

Figure 2 shows the method and variables to evaluate the viability of SST and WC definitions in highvolume airspace. In the figure the green blocks are the design variables. The tan blocks are intermediate calculations, and the blue blocks are key results. Given a desired probability of trackability and the number of measurements necessary to maintain a track, the LTV, the probability of interference at any time step can be calculated. From the probability of interference, the number of transmitting aircraft can be determined. Since the formula for the probability of interference shown in Equation (4) does not have an explicit solution for the number of aircraft, we use a root-finding method to determine this value. The total number of aircraft, the number of manned aircraft, and the maximum expected UAS density can be used to determine a maximum acceptable ADS-B transmit range. By identifying the maximum expected intruder speed and the amount of time needed to initialize a track, the trackable range can be determined. The trackable range is the maximum distance at which all intruders can be tracked with the desired probability of trackability, and thus it is the maximum possible distance-based SST definition. This definition can be mapped into a time-based definition using the maximum expected intruder speed and a time metric. We then assume that the ownship maneuvers as soon as the intruder is detected. With the minimum turn radius of the ownship, the ownship speed, and the intruder maximum speed, it is possible to determine the closest distance and time that the ownship and intruder will ever come. This distance and time are then the maximum achievable well clear distance and time respectively. The SST and WC definitions determined in this model are the maximum 
achievable definitions. Definitions larger than these are unreasonable in that the ownship cannot detect intruders at distances greater than the calculated SST and that the ownship cannot maneuver sufficiently to maintain larger distance or time WC definitions.

Airspace conditions for high-density small UAS operations are unknown. To build the model in Figure 2, it is necessary to make several assumptions about future airspace conditions. The methods and reasoning for key model details and assumptions are laid out in the following sections.

\section{Estimating Future UAS Density}

One of the first estimates necessary for the maximum SST/WC model is the density of future airspace. To determine this density, we estimated the number of small UAS that will be in Chicago, Illinois. Based on the size of Chicago, future small UAS estimates, and manned aircraft operations, the anticipated density of transmitting aircraft becomes 14.5 aircraft $/ \mathrm{nmi}^{3}$, and the density of small UAS is 13 aircraft $/ \mathrm{nmi}^{3}$ [6].

\section{Determining an Appropriate Transmit Range}

Another important aspect of the model in Figure 2 is the method used to determine an appropriate ADS-B transmit range for small UAS. In this method we first look to the probability of trackability and the limiting tracking variable. The probability of interference for a given probability of trackability can be calculated with the relationship given in Section 3 by

$$
P(\text { Interference })=\sqrt[L T V]{(1-P(\text { Trackability }))}
$$

where $L T V$ is the limiting tracking variable. With the probability of interference, it is necessary to determine the number of transmitting aircraft that lead to this probability of interference. Due to the lack of an explicit equation for the number of aircraft from the probability of interference, we determine the number of transmitting aircraft with a bisection root-finding method. With the number of aircraft and the previously determined density, the transmit range can be identified. Since the number of manned aircraft is assumed to be constant regardless of UAS transmit range, we subtract the number of manned aircraft from the total number of transmitting aircraft. This results in the number of UAS that can be transmitting within range of the ownship. Due to the fact that the UAS are assumed to operate below $400 \mathrm{ft}$, the transmit range is determined by calculating the radius of a disk that is $400 \mathrm{ft}$ thick. This transmit range is then the maximum acceptable range for ADS-B transmissions that will result in the desired probability of trackability.

It is important to note that this transmit range is only intended to apply to small UAS. Current FAA regulations dictate the transmit power, and consequently nominal range, of manned aircraft. Furthermore, the transmit range of small UAS will not significantly affect the ability of manned aircraft to track intruders. Provided that manned aircraft also have an efficient tracking method with parameters similar to those assumed for small UAS, the probability with which manned aircraft can track intruders will be very similar to the probability of trackability specified for small UAS.

\section{Calculating a Maximum Self-Separation Threshold}

The maximum self-separation threshold can be determined from the maximum tracking range. The maximum tracking range can be determined by taking the maximum acceptable transmit range and reducing it by the distance, and implicitly the time, required to initialize a track of the fastest expected target. Furthermore, the maximum tracking range is the maximum range at which tracking of all intruders can be guaranteed to the desired probability of trackability. Since this is the range at which essentially all targets can be tracked, it is reasonable to make this range the maximum self-separation threshold distance. Larger definitions of the SST are superfluous in that the ownship cannot reliably detect intruder small UAS at that range. In detailing the value for the maximum self-separation threshold, a time-based definition must also be considered. Using the ownship speed, the maximum speed of an intruder, the maximum tracking range, and an appropriate time metric, a time-based SST definition can be calculated from the distance-based SST definition.

\section{Calculating a Maximum Well Clear Definition}

With a maximum possible distance and time-based SST definition, it is possible to define a maximum possible WC definition. The maximum WC definition can be determined by calculating the minimum distance and 
time between the ownship and intruder over the course of an evasive maneuver by the ownship. If the WC definition is smaller that this minimum distance or time, then the ownship will not be able to maintain WC.

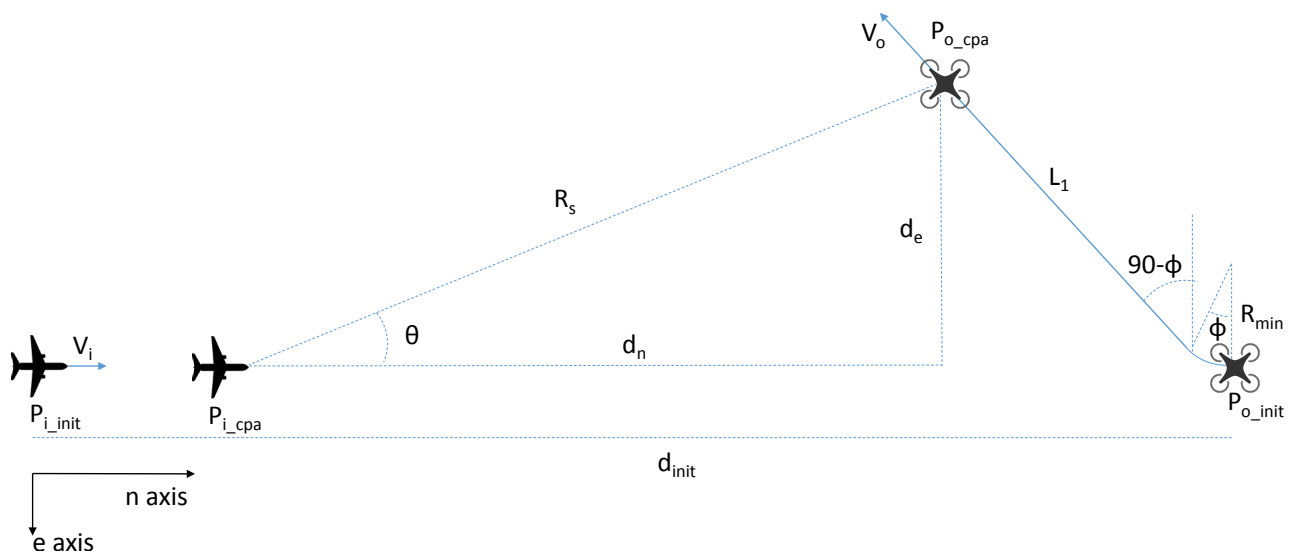

Figure 3. A parameterized maneuver by an ownship to maintain self-separation from a head-on intruder.

Figure 3 shows the geometry of an evasive maneuver in a head-on collision scenario. The ownship, represented by a multirotor, turns as tightly as possible and then continues on in a straight line. The intruder maintains a straight path as though it were oblivious to the multirotor. In Figure $3, d_{\text {init }}$ is the distance between the two aircraft at the time of the maneuver. $\phi$ is the degree by which the ownship changes course, and $R_{\min }$ is the minimum turn radius achievable by the ownship. $d_{n}$ and $d_{e}$ are the distance between the intruder and ownship at the closest point of approach in the $n$ and $e$ directions respectively. $P_{i}$ and $P_{o}$ are the positions of the intruder and ownship. Finally, $R_{s}$ and $\theta$ are the closest point of approach and the angle as shown in the figure.

\section{Determining the Minimum Distance Between an Intruder and Maneuvering Ownship}

In identifying the minimun distance between the ownship and the intruder, the starting distance between the two aircraft, $d_{\text {init }}$, is the maximum SST from the previous section. The initial positions and velocities of the ownship and intruder are also known by virtue of using ADS-B as the sensor. As a result of these known values, the first step in determining the minimum distance between the ownship and the intruder is to identify the minimum turn radius of the ownship [6]. This can be shown to be

$$
R_{\min }=\frac{V_{o}^{2}}{g \tan \phi}
$$

Thus Equation (7) shows that the minimum turn radius for a multirotor is identical to the coordinated turn equation for fixed-wing aircraft where $V_{o}$ is the velocity of the ownship. The rest of the analytic solution to the minimum closest point of approach is an extension of work done to determine the necessary sensing range for a small UAS.

Taking $P_{i_{-} \text {cpa }}$ as the origin, the closest point of approach between the two aircraft shown in Figure 3 is

$$
0=\frac{\left(R_{s} \cos \theta, R_{s} \sin \theta\right)-(0,0)}{\left\|\left(R_{s} \cos \theta, R_{s} \sin \theta\right)-(0,0)\right\|}-\left[\left(-V_{o} \sin \left(\frac{\pi}{2}-\phi\right), V_{o} \cos \left(\frac{\pi}{2}-\phi\right)\right)-\left(V_{i}, 0\right)\right] .
$$

Solving for $\tan \theta$ results in

$$
\begin{aligned}
\tan \theta & =\frac{V_{o} \cos \phi+V_{i}}{V_{o} \sin \phi} \\
& =\cot \phi+\frac{V_{i}}{V_{o} \sin \phi}
\end{aligned}
$$


Using the relationships shown in Figure 3,

$$
\begin{aligned}
t_{a r c} & =\frac{\phi R_{\min }}{V_{o}} \\
t_{l} & =\frac{L_{1}}{V_{o}} \\
\tan \theta & =\frac{d_{e}}{d_{n}} \\
d_{n} & =d_{\text {init }}-\left(t_{\text {arc }}+t_{l}\right) V_{i}-R_{\min } \sin \phi-L_{1} \cos \phi \\
d_{e} & =R_{\text {min }}(1-\cos \phi)+L_{1} \sin \phi .
\end{aligned}
$$

Using these equations and Equation (9),

$$
\frac{V_{o} \cos \phi+V_{i}}{V_{o} \sin \phi}=\frac{R_{\min }(1-\cos \phi)+L_{1} \sin \phi}{d_{\text {init }}-\left(\phi R_{\min }+L_{1}\right) \frac{V_{i}}{V_{o}}-R_{m i n} \sin \phi-L_{1} \cos \phi} .
$$

Solving for $L_{1}$ yields

$$
L_{1}=\frac{\left(\frac{V_{o} \cos \phi+V_{i}}{V_{o} \sin \phi}\right)\left(d_{i n i t}-\phi R_{\min }\left(\frac{V_{i}}{V_{o}}\right)-R_{\min } \sin \phi\right)-R_{\min }(1-\cos \phi)}{\left(\frac{V_{o} \cos \phi+V_{i}}{V_{o} \sin \phi}\right)\left(\frac{V_{i}}{V_{o}}\right)+\left(\frac{V_{o} \cos \phi+V_{i}}{V_{o} \sin \phi}\right) \cos \phi+\sin \phi} .
$$

By using in the value for $L_{1}$ to solve for $d_{n}$ and $d_{e}$ using Equations (14) and (15), the closest point of approach between the two aircraft can be calculated with the Euclidean distance formula,

$$
R_{s}=\sqrt{d_{n}^{2}+d_{e}^{2}}
$$

This result, $R_{s}$, is then the minimum distance between a straight-line intruder and a maneuvering ownship that maintains its speed and changes is course by $\phi$.

\section{Determining the Minimum Time Between an Intruder and Maneuvering Ownship}

With the minimum distance between an intruder and maneuvering ownship, it is necessary to determine how close the intruder will come to the ownship in terms of time. The time metric that we use is $\tau_{D M O D}$. This is the metric used in TCAS and it is a robust time metric. As the formula for calculating $\tau_{D M O D}$ is non-differentiable there is no analytical solution to the minimum time metric. We assume that the ownship maneuvers as shown in Figure 3. As previously stated, this is a function of the intruder speed $V_{i}$, the ownship speed $V_{o}$, the maximum detection distance $R_{\text {init }}$, the course change $\phi$, and the ownship minimum turn radius $R_{\text {min }}$. To calculate the minimum time between the ownship and intruder, we use a simulation-based method to model the motion of both aircraft over the course of the encounter. Figure 3 illustrates the worst-case, head-on scenario considered in this simulation. The intruder position, $\left(p_{i_{-} n}, p_{i_{-} e}\right)$, is modeled by

$$
\begin{aligned}
& \dot{p}_{i_{-} n}=v_{i_{-} n} \\
& \dot{p}_{i_{-} e}=v_{i_{-} e} .
\end{aligned}
$$

The ownship position, $\left(p_{o_{-} n}, p_{o_{-} e}\right)$, can be modeled by

$$
\begin{aligned}
\dot{p}_{o_{-} n} & =v_{o \_n} \\
\dot{p}_{o_{-} e} & =v_{o_{-} e}
\end{aligned}
$$

where

$$
\begin{aligned}
& v_{i_{-} n}=-V_{i} \\
& v_{i_{-} e}=0
\end{aligned}
$$




$$
\begin{aligned}
v_{o_{-} n} & = \begin{cases}V_{o} \sin (\theta) & 0 \leq \theta \leq \frac{\pi}{2} \\
V_{o} & \frac{\pi}{2}<\theta\end{cases} \\
v_{o_{-} e} & = \begin{cases}V_{o} \cos (\theta) & 0 \leq \theta \leq \frac{\pi}{2} \\
0 & \frac{\pi}{2}<\theta\end{cases} \\
\dot{\theta} & =\frac{V_{o}}{R_{\text {min }}} .
\end{aligned}
$$

From the intruder and ownship positions and velocities, it is possible to calculate a relative position and velocity at a given time step. These are given by

$$
\begin{aligned}
& p_{r}=\left(p_{i_{-} n}, p_{i_{-} e}\right)-\left(p_{o_{-} n}, p_{o_{-} e}\right) \\
& v_{r}=\left(v_{i_{-} n}, v_{i_{-} e}\right)-\left(v_{o_{-} n}, v_{o_{-} e}\right) .
\end{aligned}
$$

The minimum time between the two aircraft over the course of the maneuver is the minimum value of time metric from the set of time metrics calculated over the course of the simulation. This value is then the maximum possible well clear time definition. The maximum possible well clear distance is the minimum distance between the intruder and ownship in this worst-case scenario. Thus the maximum achievable well clear distance is the distance calculated in Equation (18).

\section{Lower WC Bound Analysis Method}

The minimum WC boundary for small UAS is key to determining a WC boundary for small UAS. The method to determine the minimum WC limit is similar to that used to find the maximum boundary.

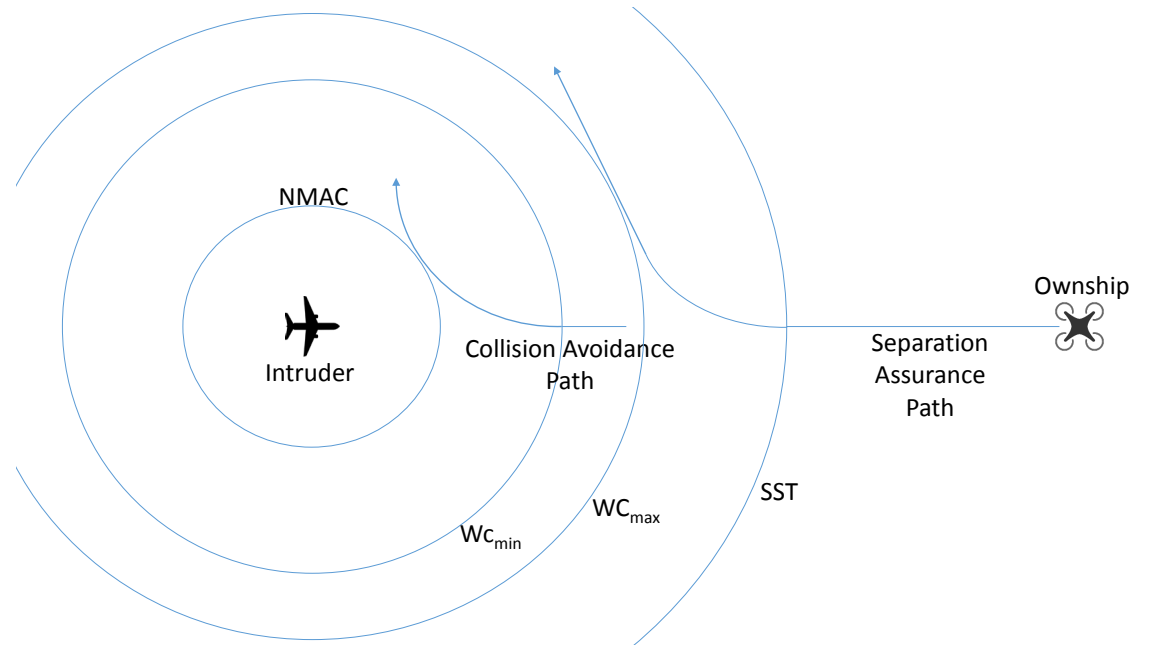

Figure 4. The methodology used to determine both the upper and lower WC bounds.

Figure 4 shows the methodology used to determine both the upper and lower WC bounds. As previously explained the upper bound is determined by the maximum distance that a maneuvering ownship can maintain from an intruder in the presence of ADS-B frequency congestion. The lower WC bound is determined by assuming that the ownship does not manuever when it first detects the intruder. Rather the ownship waits to manuever until it is just barely able to avoid the intruder NMAC volume through its maneuver. In other words the minimum WC boundary is closest that two aircraft can get before an NMAC is guaranteed. This section focuses on calculating the lower WC boundary.

In calculating the lower WC boundary there are two cases to consider. The first case occurs when $R_{\min }<R_{N M A C}$, and the second case occurs when $R_{\min }>R_{N M A C}$. These two cases each yield different equations. The minimum $\mathrm{WC}$ for case one is

$$
d_{\min W C}=\frac{1}{V_{o}}\left(\frac{V_{o}^{2}\left(V_{o}+(\pi / 2-1) V_{i}\right.}{g \tan \phi}+R_{N M A C} \sqrt{V_{o}^{2}+V_{i}^{2}}\right) .
$$


The equation for case two is

$$
\begin{aligned}
d_{\text {min } W C}= & \sqrt{R_{N M A C} \sin \theta\left(2 R_{\min }-R_{N M A C} \sin \theta\right)} \\
& +\frac{V_{i}}{V_{o}}\left(R_{\text {min }} \cos ^{-1}\left(\frac{R_{\text {min }}-R_{N M A C} \sin \theta}{R_{\min }}\right)\right)+R_{N M A C} \cos \theta
\end{aligned}
$$

where $\theta$ is calculated using a bisection method on

$$
0=\frac{V_{o} \sin \theta \sqrt{R_{N M A C} \sin \theta\left(2 R_{\min }-R_{N M A C} \sin \theta\right)}}{R_{\min }}-\left(\frac{V_{o}\left(R_{\min }-R_{N M A C} \sin \theta\right)}{R_{\min }}+V_{i}\right) \cos \theta
$$

Equations (19) and (20) calculate the distance necessary to ensure that an ownship can maneuver sufficiently to avoid the NMAC volume of an intruder. Thus, $d_{\min W C}$ is the minimum WC distance for small UAS. The time-based minimum WC definition is determined in the same way as the maximum WC definition, but $R_{N M A C}$ is used in the $\tau_{D M O D}$ equation rather than the maximum WC definition.

\section{Results}

\section{A. ADS-B Congestion Simulation Results}

To test and demonstrate the probability of interference and the probability of trackability, we present two key plots. The first plot illustrates the probability of interference. The second plot shows the results of a simulation in which we simulate the interference of individual intruders as we gradually increase the ADS-B transmit range of the intruders.

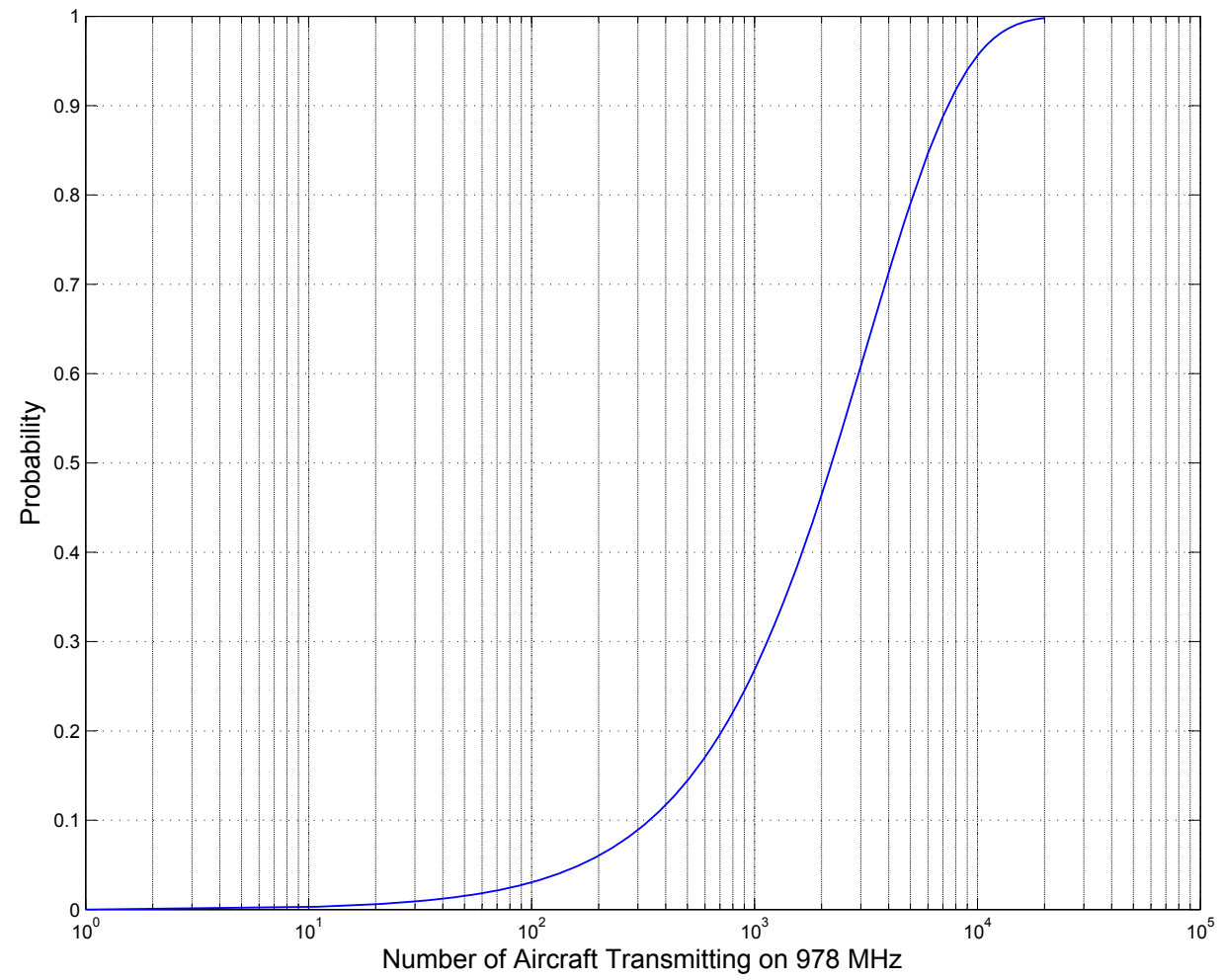

Figure 5. Probability of interference as a function of the number of transmitting aircraft.

The first plot, shown in Figure 5, shows the probability of interference as a function of the number of transmitting aircraft. This plot provides important perspective on the number of ADS-B-equipped aircraft that would lead to significant levels of interference. While the values represented on the horizontal axis 
of Figure 5 are large, it is possible that future airspace conditions would have several thousand aircraft operating at the same time in the same region.

Figure 6 presents the results of a simulation that highlights the importance of estimating intruder states and the limitations that increased transmit range imposes on intruder visibility. To create Figure 6, we generate a large set of intruders uniformly distributed over a cubical airspace with a set density. Then we vary the ADS-B transmit range linearly. For each transmit range, we determine the number of intruders that are within range and calculate the probability of interference. With the probability of interference we sample a uniform distribution to determine which intruders have been interfered with and are thus invisible at that time step. We also calculate which intruders we are tracking. With the list of total intruders, intruders in range, visible intruders, and the tracked intruders, we calculate key statistics to determine the visibility and trackability of the intruders.

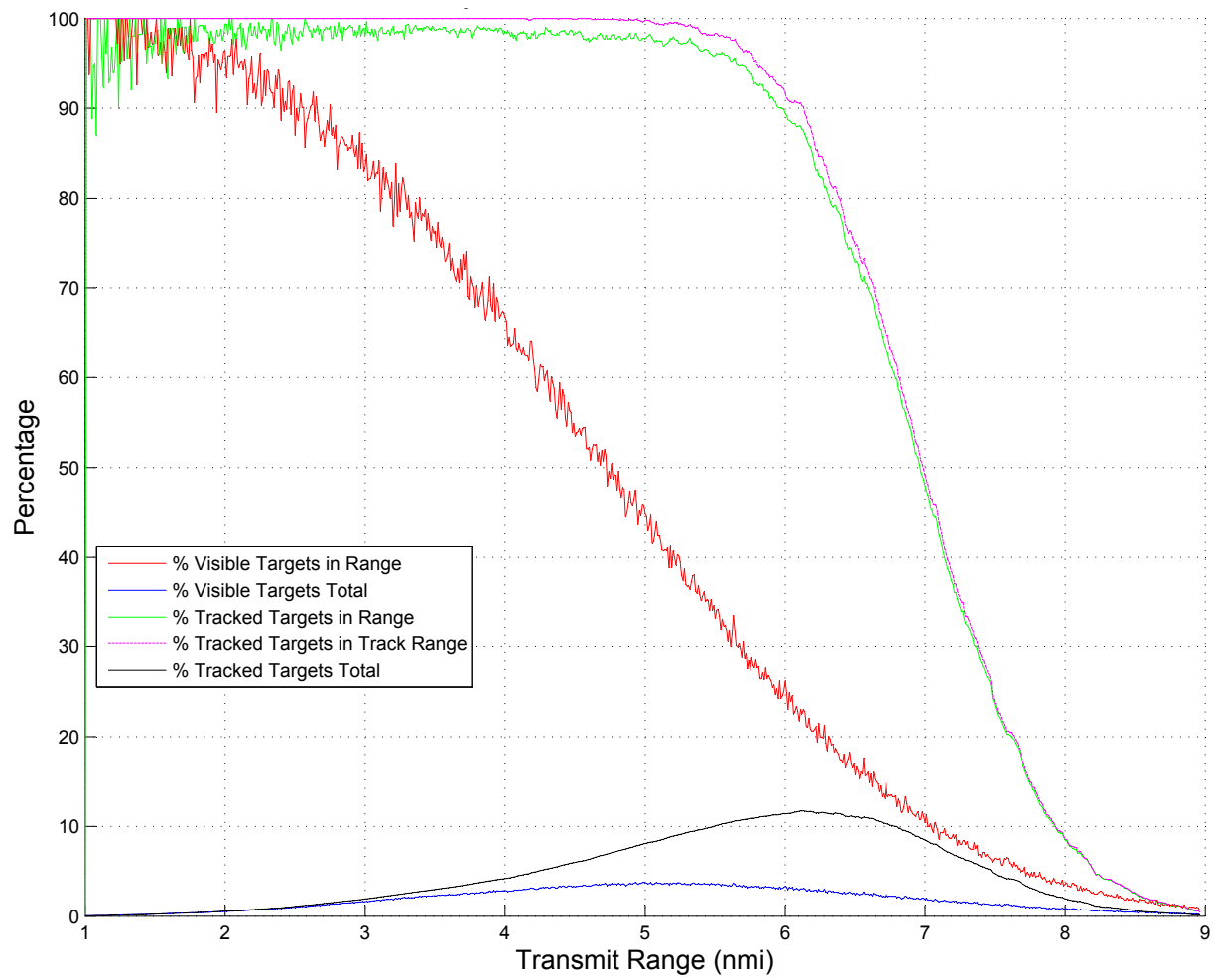

Figure 6. Influence of increasing range on the probability of interference and the probability of trackability. It also demonstrates the impact of those metrics on the overall detectability of intruders.

In Figure 6 there are five lines. The red line shows the percentage of targets that are within range that are also visible at a given transmit range. The green line shows the percentage of targets that are in range and trackable for a given transmit range. It is interesting to note that the green line is always less than one, even for very short transmit ranges. This is a result of intruders moving into and out of the visible region. As an intruder enters the visible region, it takes several time steps to initialize a track. During that initialization period the aircraft is in range, but it is not trackable. To determine a range at which all intruders should be trackable, we define a region slightly smaller than the visible region by which point all tracks should have been able to initialize. The pink line shows the percentage of intruders within the smaller region that are trackable. As would be expected the pink line remains a 100\% until the interference becomes very high. The blue and black lines show the percentage of total intruders that are in the simulation, within range and outside the range, that are visible and trackable respectively.

Figure 6 illustrates several important principles of ADS-B interference. As previously mentioned tracking of intruders significantly increases the amount of interference that an ADS-B-based DAA system can tolerate. This can be seen in the disparity between the red and green lines. The red line drops below $90 \%$ visibility at approximately $2.5 \mathrm{nmi}$. The green line, however, is able to maintain $90 \%$ trackability until $6 \mathrm{nmi}$. This is a significant increase in the ability of an ownship to detect intruders. Essentially at a transmit range of 
$2.5 \mathrm{nmi}$, a DAA system without intruder tracking is only capable of knowing the position and velocity of $90 \%$ of intruders with in the $2.5 \mathrm{nmi}$ range. A DAA system with intruder tracking, on the other hand, is capable of knowing the position and velocity of $90 \%$ of the intruders upto a transmit range of $6 \mathrm{nmi}$.

Another key takeaway is that once an intruder enters the visible range, there is an initialization period before that intruder can be accurately tracked. This fact is seen in the difference between the pink and green lines. While the initialization distance for new tracks is very small compared to the transmit range, the difference between the transmit range and the tracking range has important implications for analyses of the necessary transmit range to allow for conflict/collision avoidance.

One of the most important takeaways from Figure 6 is the distinctive peak in both the blue and black lines. Initially, it seems that as the transmit range increases the number of visible/trackable targets should also increase. Stated otherwise, the farther that the ownship can see the more intruders it can see. The peak in the blue and black lines indicate that this is not the case. As the transmit range increases, the number of transmitting aircraft in range also increases. Thus there is in reality a point at which the number of aircraft in range becomes so large that the interference rises to a level that actually reduces the visibility/trackability of the targets in range. Thus if all aircraft are transmitting ADS-B too far, then the overall visibility/trackability is reduced to levels below that of shorter transmit ranges. This is a significant result, and it shows that too much ADS-B transmit power can reduce the capability of a DAA system.

\section{B. Well Clear Definition Simulation Results}

Given the limitations of ADS-B congestion, this section shows the results of a series of calculations/simulations wherein we determine the maximum and minimum acceptable SST and WC definitions for a given set of parameters. We start by demonstrating and exercising the model presented in Figure 2. Then we couple the minimum WC definitions from Section 8 to create a well clear recommendation for small UAS. In both the maximum and minimum WC analysis we use $\tau_{D M O D}$ as the time metric. This is the metric used by both TCAS and other leading WC recommendations for larger aircraft [11]. $\tau_{D M O D}$ is a modified time to collision and is given by

$$
\tau_{D M O D}=\left\{\begin{array}{ll}
-\frac{\left(r^{2}-H M D^{2}\right)}{r \dot{r}} & C P A \leq H M D \\
\infty & C P A>H M D
\end{array} .\right.
$$

In Equation $22 r$ and $\dot{r}$ are the range and range rate between the intruder and ownship respectively. Additionally both the maximum and minimum WC definitions rely on a set of several parameters. These parameters are given in Table 1.

Table 1. Key parameters for SST and WC definition evaluation.

\begin{tabular}{ll}
\hline Parameter & Value \\
\hline Intruder Speed & $250 \mathrm{kt}$ \\
Probability of Trackability & 0.999999 \\
Message Start Opportunities & 3200 \\
UAS Density & $13.77 \mathrm{UAS} / \mathrm{nmi}^{3}$ \\
\hline UAS Type & Multirotor \\
Ownship Bank Angle & $40 \mathrm{deg}$ \\
Ownship Course Change & $90 \mathrm{deg}$ \\
Lift-to-Weight Ratio & 1.5 \\
HMD Value & $0.65 \mathrm{nmi}$ \\
Max UAS Altitude & $0.0658 \mathrm{nmi}(400 \mathrm{ft})$ \\
Limiting Tracking Variable & 11 \\
\hline
\end{tabular}

The initial values of the parameters in Table 1 are designed to closely match the operational characteristics of current airspace. This scenario serves as a baseline with which all other calculations can be compared. Table 1 shows the baseline values of the key parameters. The upper group of parameters in Table 1 are 
those parameters that will be varied in the subsequent analysis, and the lower group of parameters are those parameters that are not expected to change.

\section{Maximum WC Model}

To demonstrate the maximum SST and WC method in Figure 2, we analyzed a WC definition proposed by the Sense and Avoid Science Research Panel (SARP) based on a rigorous set of simulations and testing [11]. The method defines the distance threshold and HMD to be $0.6583 \mathrm{nmi}(4000 \mathrm{ft})$ and the time-based threshold to be $\tau_{D M O D}=35 \mathrm{~s}$. The SST that we examine in our analysis was proposed by NASA Ames Research Center and is $\tau_{D M O D}=90 \mathrm{~s}$ and distance and HMD of $0.6583 \mathrm{nmi}(4000 \mathrm{ft})$ [10].

To provide perspective on the ability of all types of UAS to maintain the proposed SST and WC definitions, we sequentially the compute the model for several different ownship speeds. Additionally we explore the sensitivity of key parameters by varying them individually and observing the effect on the maximum SST and WC definitions. The initial scenario uses the parameter values shown in Table 1 which are designed to closely match the operational characteristics of current airspace.
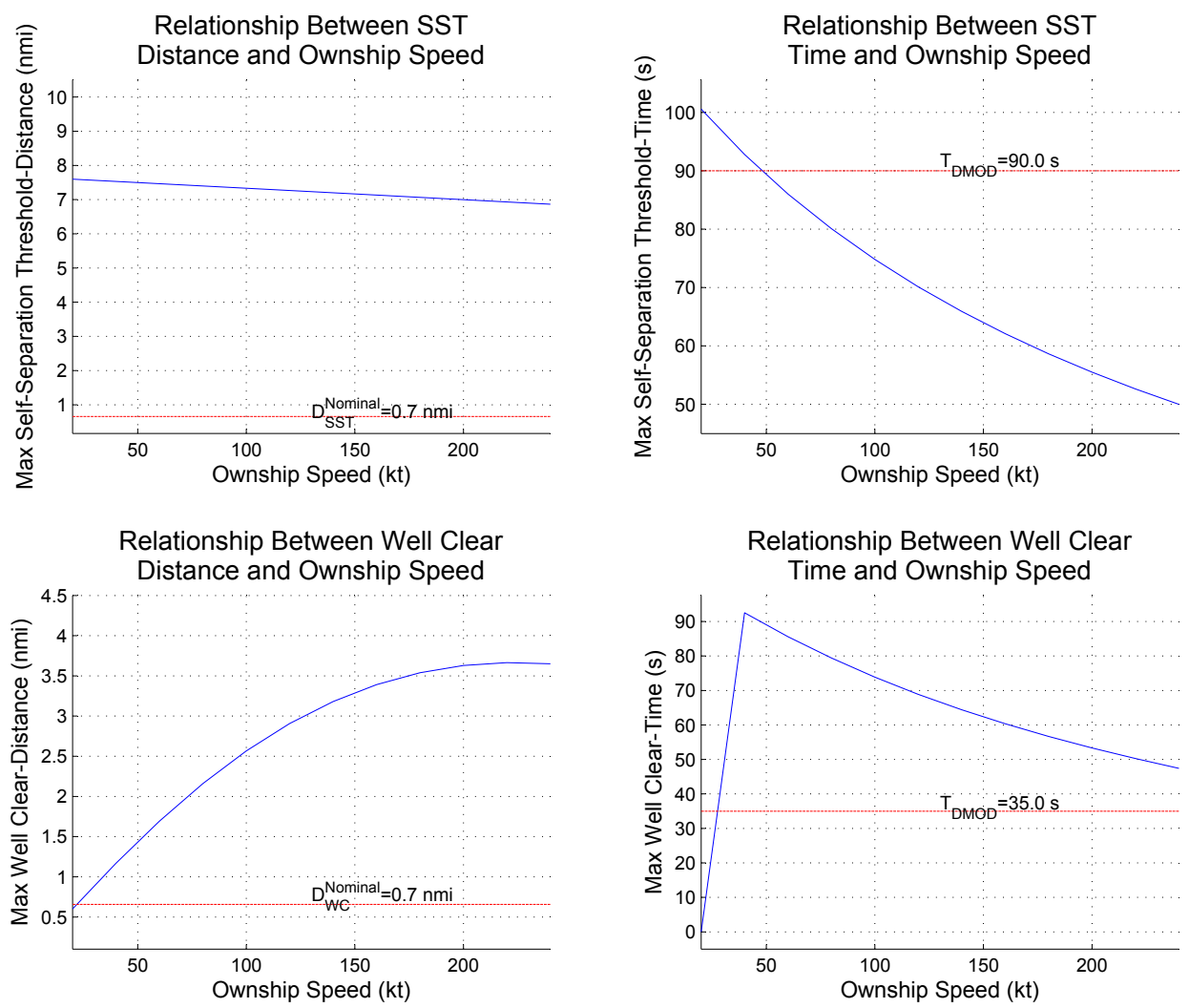

Figure 7. Minimum achievable SST and WC definitions for current airspace conditions.

Figure 7 shows the minimum achievable SST and WC definitions. The red line in each plot represents the nominal value of the definition $[10,11]$. The top left plot shows the maximum SST distance definition. As is clear from the figure, the ownship is able to detect intruders far before they cross the distance-based SST. As the speed of the ownship increases, the detection range decreases slightly due to the increased distance necessary to initialize a track. The top right plot shows the value of $\tau_{D M O D}$ when the intruders are detected. From this plot it is very clear that the $90 \mathrm{~s}$ time-based SST is much too large. For all ownship speeds greater than approximately $35 \mathrm{kts}$, the maximum achievable time-based SST is less than the nominal SST definition. Thus when the intruders are detected they will already have crossed the time-based SST. The bottom left plot shows the maximum achievable distance-based WC definition. From the plot it is clear that nearly all ownship speeds are able to maintain the nominal WC distance, but ownships moving at $20 \mathrm{kts}$ are unable to maneuver in time. The bottom right plot reinforces this. Ownships moving at $20 \mathrm{kts}$ are unable to maneuver 
sufficiently to achieve the required horizontal miss distance. Thus the aircraft comes too close in terms of both time and distance.

Overall Figure 7 indicates that the current airspace regulations are not suitable for high-density small UAS operations. Small, relatively slow-moving UAS, such as the DJI Phantom 1, are unable to maneuver fast enough to maintain the proposed SST and WC definitions. Furthermore, from the top right plot it is clear that the maximum time-based SST that is viable for all ownship speeds is $\tau_{D M O D}=46 \mathrm{~s}$. For a $\mathrm{WC}$ time-based definition that is $\tau_{D M O D}=35 \mathrm{~s}$, this is very little maneuver time. Interestingly the same high-speed ownships for whom the time-based SST must be lowered to $\tau_{D M O D}=46 \mathrm{~s}$ are able to maintain the $\tau_{D M O D}=35 \mathrm{~s}$ time-based WC definition. Thus the time-based SST definition is too small for the slow UAS and too large for the fast UAS. Changing it would result is an equally unacceptable solution regardless of the direction of change. This shows that the time-based SST definition is not to blame. It is reasonable to deduce that the underlying problem is not the SST and WC definitions, but rather that the airspace regulations and conditions are unsuitable to high-density small UAS operations.
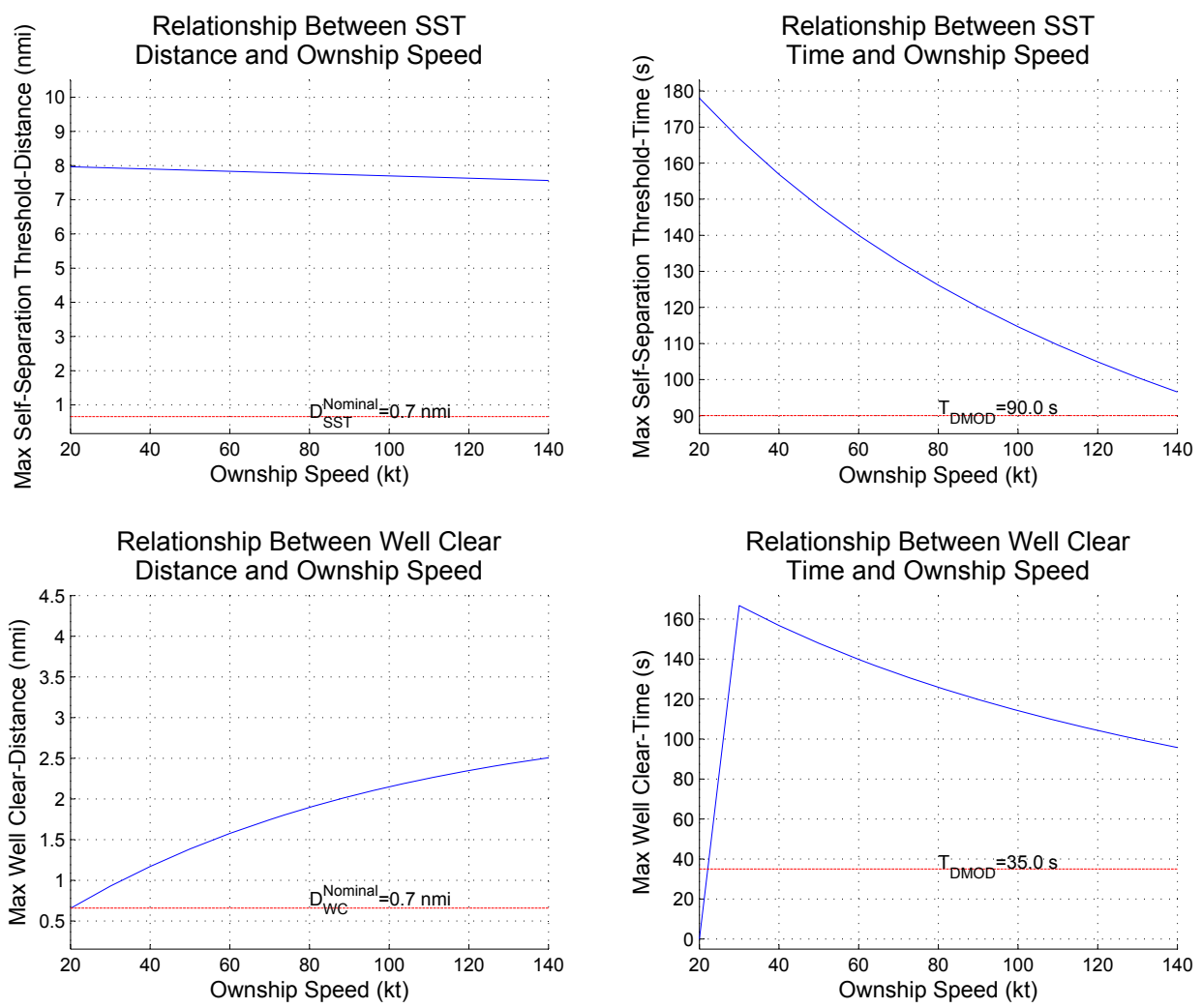

Figure 8. Achievable SST and WC definitions as a result of alterations to several airspace conditions and regulations in accordance with Amazon and Google UAS integration vision.

It is important to note that the results shown in Figure 7 are for an ownship that maneuvers as aggressively as possible as soon as the intruder comes into view. Such a demand on the ownship undermines much of the intent behind self-separation. Maintaining well clear is typically understood to imply a gradual change in flight path to ensure a safe distance between aircraft. Demanding that a small UAS turn as sharply as physics will allow from its original path and fly perpendicular to that path as fast as it can is much more indicative of a collision avoidance maneuver. Such maneuvers would seriously interfere with the intended mission of a small UAS, and may be intolerable. Additionally it is very unlikely that small, slow-moving UAS will be operating in the same vicinity as manned aircraft that are traveling at $250 \mathrm{kts}$. In accordance with Google's [17] and Amazon's (amazon.com/primeair) published vision for small UAS operation, it is reasonable to expect that small UAS will only encounter relatively slow manned aircraft such as police and medical helicopters. In Amazon's plan they envision a "High-Speed Transit" region that is between $200 \mathrm{ft}$ and $400 \mathrm{ft}$ above ground level. It is in this region where small UAS would encounter manned aircraft. In such a region, very slow moving aircraft such as the DJI Phantom 1, which has a max speed of $20 \mathrm{kts}$, would 
not be permitted.

As a result of these expectations, the maximum WC model is adjusted to have a maximum intruder speed of $140 \mathrm{kts}$ and an ownship course change of 40 degrees. Figure 8 shows the maximum achievable SST and WC definitions for this scenario. From the figure it is clear that while the slowest UAS are not able to maintain WC, almost all UAS are able to maintain WC from intruders traveling at $140 \mathrm{kts}$. Furthermore both the distance and time-based SST definitions are achievable. As a result of Figure 8, the maximum SST definition for small UAS in congested airspace should be $7.56 \mathrm{nmi}$ in distance or $\tau_{D M O D}=96.5 \mathrm{~s}$. The maximum WC definition under the same conditions should be $0.658 \mathrm{nmi}$ of distance and $\tau_{D M O D}=95.7 \mathrm{~s}$. Thus the definition recommended by SARP is achievable under the proposed low-speed, low-altitude airspace.
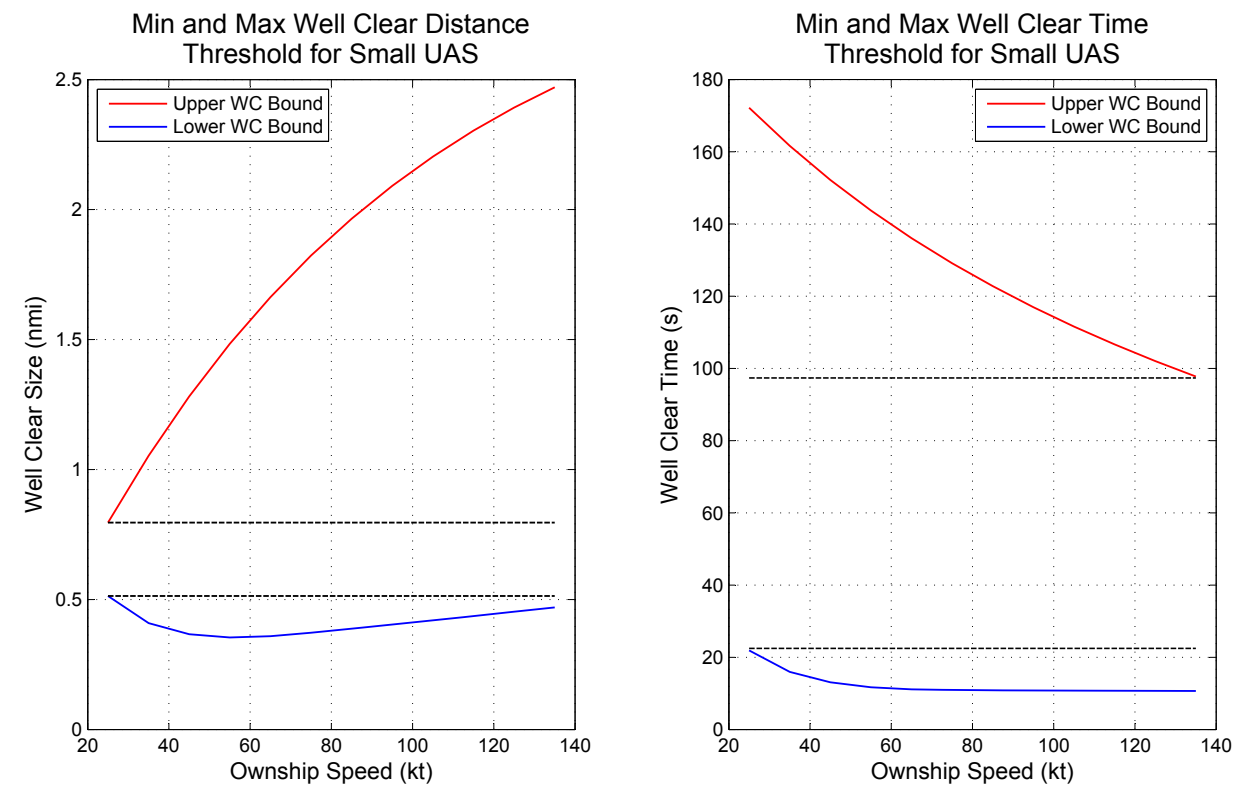

Figure 9. Minimum and maximum WC definitions.

\section{Minimum WC Model}

The maximum SST and WC definitions provide valuable insight on what is achievable for small UAS. To make a recommendation for WC for small UAS, however, a minimum WC criterion is also necessary. The modified equations for the minimum detection range discussed in Section 8 provide that lower bound. As before the minimum WC definition was calculated for multiple ownship speeds to show the WC definition for a range of ownship types.

Figure 9 shows both the upper and lower WC bound as the ownship speed varies. The red lines are the upper bound. Although the upper bound varies at different ownship speeds, the true upper bound is the minimum point on the red line. This ensures that the maximum WC bound is viable for all ownships across the range of speeds. The blue line is the lower bound. In terms of distance the maximum point on the blue line is the true lower bound for all ownship speeds. For time, however, the minimum point on the blue line is the lower WC time bound. The true bounds are shown by the black dotted line.

The figure provides several key insights. First, low speed ownships require the most distance to maneuver. This is because they are moving sufficiently slowly that they are unable to clear the path of the intruder before an NMAC or loss of WC occurs. Secondly, the figure indicates that appropriate WC distance definitions for small UAS in the presence of slow-moving manned aircraft are on the order of $0.5 \mathrm{nmi}(3038 \mathrm{ft})$. This is larger than anticipated, and it is a valuable anchor point to inform the discussion of WC for small UAS. Third, and most importantly, the combination of an upper and lower WC bound is a basis for recommending a WC definition for small UAS.

On that basis we recommend that the WC definition for small UAS be $0.527 \mathrm{nmi}(3200 \mathrm{ft})$ or $\tau_{D M O D}=25 \mathrm{~s}$. This definition falls between the boundaries shown in Figure 9. As additional data, such as small UAS flight patterns, become available, these bounds and consequently the recommendation can be refined. In the 
current absence such data, this recommendation is a key step to understanding operationally feasible SST and WC definitions for small UAS.

\section{Conclusions}

In summary the analyses and simulations presented here demonstrate the bandwidth limitations of $978 \mathrm{MHz}$ ADS-B and the effect of those limitations on a well clear definition for small UAS in high-density, ADS-B-based airspace. Furthermore it provides a tool with which SST and WC definitions can be evaluated with respect to their feasibility for future high-density, ADS-B-equipped airspace. Most importantly the recommendation for well clear for small UAS is a key step to integrating small UAS into the national airspace. As the body of knowledge of the operational environment of small UAS grows, the proposed well clear recommendation can be refined. Until then the recommendation presented here is a key starting point. Overall the approach, methods, and recommendations here are a key contribution to defining well clear and self-separation thresholds for future small UAS-dense airspace.

\section{Acknowledgments}

This work has been funded in large part by the Center for Unmanned Aircraft Systems, a National Science Foundation-sponsored industry/university cooperative research center (I/UCRC). Additional funding was provided by the Utah NASA Space Grant Consortium.

\section{References}

${ }^{1} 112$ th Congress, "FAA Modernization and Reform Act of 2012," 2012.

${ }^{2}$ Hottman, S., Hansen, K., and Berry, M., "Literature Review on Detect, Sense, and Avoid Technology for Unmanned Aircraft Systems," Tech. Rep. September, 2009.

${ }^{3}$ Federal Aviation Administration, "Subchapter F - Air Traffic and General Operating Rules," 2015.

${ }^{4}$ White, D., "Google and Chipotle Are Testing Drone Burrito Delivery at Virginia Tech," Time, Sept. 2016.

${ }^{5}$ George, S., "Concepts of use for UAS Sense and Avoid Equipment," Tech. rep., 2009.

${ }^{6}$ Duffield, M. O., Mclain, T. W., Beard, R. W., and Ning, S. A., Automatic Dependent Surveillance-Broadcast for Detect and Avoid on Small Unmanned Aircraft, Thesis, Brigham Young University, 2016.

${ }^{7}$ Moody, C. and Strain, R., "Implementation Consideration for Automatic Dependent Surveillance - Broadcast on Unmanned Aircraft Systems," AIAA Infotech@Aerospace Conference, American Institute of Aeronautics and Astronautics, Reston, Virigina, April 2009, pp. 1-8.

${ }^{8}$ Federal Aviation Administration, "Automatic Dependent Surveillance- Broadcast (ADS-B) Out Performance Requirements To Support Air Traffic Control (ATC) Service," 2010.

${ }^{9}$ Munoz, C., Narkawicz, A., Chamberlain, J., Consiglio, M., and Upchurch, J., "A Family of Well-Clear Boundary Models for the Integration of UAS in the NAS," 14th AIAA Aviation Technology, Integration, and Operations Conference, Atlanta, GA, 2014, pp. 1-16.

${ }^{10}$ Johnson, M., Mueller, E. R., and Santiago, C., "Characteristics of a Well Clear Definition and Alerting Criteria for Encounters between UAS and Manned Aircraft in Class E Airspace," 11th USA/Europe Air Traffic Management Research and Development Seminar, Lisbon, Portugal, 2015.

${ }^{11}$ Cook, S. P., Brooks, D., Cole, R., Hackenburg, D., and Raska, V., "Defining Well Clear for Unmanned Aircraft Systems," AIAA Infotech@Aerospace, No. January, Kissimmee, FL, 2015, pp. 1-20.

${ }^{12}$ Upchurch, J., Munoz, C., Narkawicz, A., Chamberlain, J., and Consiglio, M., "Analysis of Well-Clear Boundary Models for the Integration of UAS in the NAS," Tech. Rep. June, NASA Langley Research Center, Hampton, VA, 2014.

${ }^{13}$ Mullins, M., Holman, M., Foerster, K., Kaabouch, N., and Semke, W., "Dynamic Separation Thresholds for a Small Airborne Sense," Guidance, Navigation, and Control, Boston, MA, 2013, pp. 1-6.

${ }^{14}$ Weibel, R. E., Edwards, M. W. M., and Fernandes, C. S., "Establishing a Risk-Based Separation Standard for Unmanned Aircraft Self Separation," USA/Europe Air Traffic Management Research \& Development Seminar, No. June, Berlin, Germany, 2011, pp. 14-17.

${ }^{15}$ RTCA, "Minimum Operational Performance Standards for Universal Access Transceiver ( UAT ) Automatic Dependent Surveillance - Broadcast ( ADS-B ) DRAFT Final Review and Comment," 2009.

${ }^{16}$ Papoulis, A. and Pillai, S. U., Probability, Random Variables, and Stochastic Processes, McGraw-Hill New York, 4th ed., 2002.

${ }^{17}$ Google Inc., "Google UAS Airspace System Overview," . 